\title{
Time-dependent wave splitting and source separation
}

\author{
Marcus J. Grote ${ }^{\mathrm{a}}$, Marie Kray ${ }^{1 \mathrm{a}}$, Frédéric Nataf ${ }^{\mathrm{b}, \mathrm{c}, \mathrm{d}}$, Franck Assous ${ }^{\mathrm{e}}$ \\ ${ }^{a}$ Department of Mathematics and Computer Science, University of Basel, Spiegelgasse 1, \\ CH-4051 Basel, Switzerland, \{marcus.grote, marie.kray\}@unibas.ch \\ ${ }^{b}$ CNRS, UMR 7598, Laboratoire Jacques-Louis Lions, F-75005, Paris, France \\ ${ }^{c}$ UPMC Univ Paris 06, UMR 7598, Laboratoire Jacques-Louis Lions, F-75005, Paris, \\ France, nataf@ann.jussieu.fr \\ ${ }^{d}$ INRIA Rocquencourt, Alpines, BP 105, 78153 Le Chesnay Cedex, France \\ ${ }^{e}$ Department of Mathematics, Ariel University, 40700, Ariel, Israel, \\ franckassous55@gmail.com
}

\begin{abstract}
Starting from classical absorbing boundary conditions, we propose a method for the separation of time-dependent scattered wave fields due to multiple sources or obstacles. In contrast to previous techniques, our method is local in space and time, deterministic, and avoids a priori assumptions on the frequency spectrum of the signal. Numerical examples in two space dimensions illustrate the usefulness of wave splitting for time-dependent scattering problems.
\end{abstract}

Keywords:

Time-dependent scattering, wave splitting, source separation, absorbing boundary conditions

\section{Introduction}

As natural information carriers, acoustic, electromagnetic and elastic waves are ubiquitous for probing an unknown medium to determine its internal geometric or material properties. When the incident probing wave encounters an inhomogeneity, it generates a scattered wave which carries information about the obstacle across the background medium. From that scattering data, usually recorded at remote sensors, the nature, location and

\footnotetext{
${ }^{1}$ corresponding author
} 
shape of the obstacle buried inside the medium can be recovered, as in seismic full waveform inversion, medical imaging, and nondestructive testing. Clearly, that information is readily available by subtraction of the incident wave from total field measurements. However, if the location, spatial distribution or time dependence of the original source are not precisely known, or other undesired sources interfere with the signal, extraction of the scattered field of interest becomes non-trivial, though it remains essential for any subsequent inversion.

Blind source separation in signal processing [7], for instance, attempts to separate the input signals from their recorded mixture, much like in the well-known "cocktail-party effect" [20] where listeners perceptually focus on a single target voice against competing sound sources. Ray-based in nature, blind source separation uses statistical tools to detect individual sources [9] or remove noise [2] from linearly superimposed signals. Other applications of source separation, also known as signal separation, include 3D virtual surround sound [31] and the development of hearing aids [25].

In transcranial ultrasonic imaging, intense ultrasound pulses induce a single cavitation bubble whose collapse generates a small shock wave then recorded by a standard ultrasound imaging array [26]; clearly, the bubble's time signature is never precisely known. Similarly, the detection of individual free-floating and targeted microbubbles of an ultrasound contrast agent is critical for quantifying the amount of bubbles in the tissue [23]. Yet another application is photoacoustic imaging, where laser pulses are delivered into biological tissues thereby leading to transient thermoelastic expansion and subsequent ultrasonic emission. Again, the quantity of interest is the time signature of the laser induced pressure wave generated by an unknown source [29].

When two or more obstacles are present, each primary scattered wave will induce secondary scattered waves from all other obstacles, which again will induce further scattered waves, and so forth. Together with the incident wave, their superposition results in the measured total wave field. The inversion from the total wave field for multiple obstacles at once adds yet another layer of complexity to any algorithm for inverse scattering problems. Hence, if their superposition can be split into individual outgoing components, we can apply any algorithm for single inverse scattering to each scatterer separately. As a consequence, each isolated scattering problem will be smaller in size and less ill-conditioned than their total sum.

In seismic imaging, for instance, shear-wave splitting has been successfully 
used for earthquake prediction or in the petroleum industry. Indeed when a polarized elastic shear wave enters a new, anisotropic medium, it splits into two shear waves whose mutual time-delay and orientation then allows to infer the density and orientation of the cracks in the medium. In [24], Kreider uses Weston's theoretical wave splitting for stratified media [32] to reconstruct the unknown velocity function in a stratified inhomogeneous cylinder from reflection data.

There is a long history of wave splitting techniques for multiple scattering problems, especially in the frequency domain. In his classical work, Twerksy expresses multiple scattering coefficients in terms of algebraic relations which couple the multipole coefficients of isolated scatterers - see [30] for a review. More recently, Grote and Kirsch [14] used wave splitting to derive nonreflecting boundary conditions for multiple scattering problems. Similarly, Acosta [1] formulated on-surface radiation conditions for multiple scattering. In [8], Ben Hassen, Liu and Potthast split the far-field pattern using integral based formulations to extend the point source method to inverse scattering of multiple obstacles. By combining the inverse Radon approximation with a Galerkin ansatz, Griesmaier, Hanke and Sylvester determine the convex scattering support of individual far-field components separately [12].

In the time domain, little work on inverse multiple scattering problems is available. In $[15,17]$, nonreflecting boundary conditions for time-dependent multiple scattering were derived, which avoid the space-time integrals involved in standard integral-based formulations. By Fourier transform in the frequency domain, Potthast, Fazi and Nelson [27] devised a filter via the point source method for time-dependent source separation.

Here we propose a method to determine the separate outgoing components of the incident and scattered wave fields for time-dependent scattering problems. In the case of two superposed wave fields, our method applies to the following three typical configurations: two distinct localized sources with unknown time history each, a single (unknown) localized source with a nearby scatterer, or two separate scatterers illuminated by a known incident wave field. In all three cases, our method permits to recover the individual outgoing components from measurements of the total scattered field at a distance. In doing so, the particular nature of the scatterer, be it an impenetrable well-defined obstacle or a penetrable localized inhomogeneity, is immaterial; only the purely outgoing character of the individual wave fields matters.

In contrast to previous work, our approach is local in space and time, 
deterministic, and also avoids any a priori assumptions on the frequency spectrum of the signal. Starting from the standard Bayliss-Turkel [5] absorbing boundary conditions, we present in Section 2 the principle of wave splitting, first introduced in [16], which we then illustrate in the simpler onedimensional case. Next, in Section 3, we present our wave splitting approach in the general two-dimensional case and also discuss its numerical implementation. Finally, in Section 4, we consider two distinct numerical experiments to illustrate the usefulness of our wave splitting approach.

\section{Wave splitting}

We consider wave scattering from two distinct scatterers, which each may contain several obstacles, inhomogeneities and nonlinearity. Both scatterers are well separated, that is we assume that we can surround them by two non-intersecting spheres $S_{1}$ and $S_{2}$ centered at $C_{1}$ and $C_{2}$, respectively see Fig. 1. In $\Omega$, the unbounded domain outside $S_{1}$ and $S_{2}$, the scattered wave field $u$ satisfies:

$$
\frac{\partial^{2} u}{\partial t^{2}}-c^{2} \Delta u=0 \quad \text { in } \Omega, \quad t>0
$$

with constant wave speed $c>0$. Moreover, we assume that $u$ is initially confined to the interior of $S_{1} \cup S_{2}$; thus, it is zero throughout $\Omega$ at time $t=0$. Then $u$ splits into two unique wave fields $u_{1}$ and $u_{2}$ as

$$
u=u_{1}+u_{2} \quad \text { in } \Omega, \quad t>0,
$$

where each $u_{k}$ is purely outgoing and satisfies (1) in the entire exterior of $S_{k}$ [15]; hence, $u_{k}$ is determined by its time-dependent (unknown) values on $S_{k}, k=1,2$. Now, let $\Gamma$ denote a surface patch or curve segment, not necessarily closed or connected, which lies inside $\Omega$. Given the time history of the scattered field $u$ on $\Gamma$, we seek to recover the two outgoing components $u_{1}$, $u_{2}$ on $\Gamma$ for all time.

Since each $u_{k}$ is outgoing outside $S_{k}$, it can be written as a progressive wave expansion in inverse powers of distance $[5,18]$ from $C_{k}$. In three space dimensions, for instance, we have

$$
u_{k}\left(t, r_{k}, \theta_{k}, \varphi_{k}\right)=\frac{1}{r_{k}} \sum_{i \geq 0} \frac{f_{k, i}\left(r_{k}-c t, \theta_{k}, \varphi_{k}\right)}{\left(r_{k}\right)^{i}}
$$




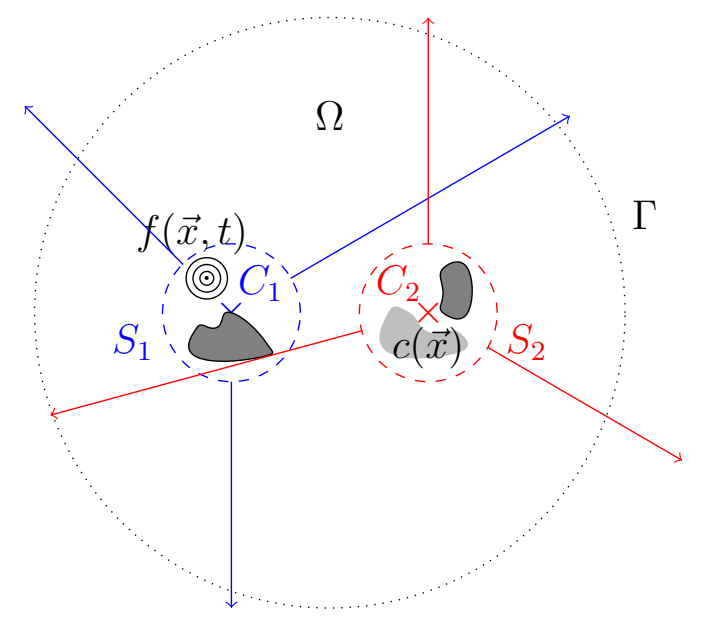

Figure 1: Two distinct scatterers and their corresponding outgoing wave fields.

where $\left(r_{k}, \theta_{k}, \varphi_{k}\right)$ denote spherical coordinates centered about the origin $C_{k}$, $k=1,2$. In two space dimensions, the progressive wave expansion of $u_{k}$ converges only asymptotically:

$$
u_{k}\left(t, r_{k}, \theta_{k}\right) \sim \frac{1}{\sqrt{r_{k}}} \sum_{i \geq 0} \frac{f_{k, i}\left(r_{k}-c t, \theta_{k}\right)}{\left(r_{k}\right)^{i}}, \quad r_{k} \rightarrow \infty,
$$

where $\left(r_{k}, \theta_{k}\right)$ denote polar coordinates centered about the origin $C_{k}, k=1,2$.

Starting from (3), (4) respectively, Bayliss and Turkel [5] derived a sequence of increasingly accurate differential operators of order $m$ that annihilate the $m$ leading order terms in either expansion above for $k=1,2$ :

$$
\begin{aligned}
& B_{k}^{m}\left[u_{k}\right]:=\prod_{\ell=1}^{m}\left(\frac{1}{c} \frac{\partial}{\partial t}+\frac{\partial}{\partial r_{k}}+\frac{2 \ell-1}{r_{k}}\right) u_{k}=O\left(\frac{1}{r_{k}^{2 m+1}}\right), \Omega \subset \mathbb{R}^{3},(5) \\
& B_{k}^{m}\left[u_{k}\right]:=\prod_{\ell=1}^{m}\left(\frac{1}{c} \frac{\partial}{\partial t}+\frac{\partial}{\partial r_{k}}+\frac{4 \ell-3}{2 r_{k}}\right) u_{k}=O\left(\frac{1}{r_{k}^{2 m+\frac{1}{2}}}\right), \Omega \subset \mathbb{R}^{2} .(6)
\end{aligned}
$$

By setting

$$
B_{k}^{m}\left[u_{k}\right]=0,
$$

each operator yields an absorbing boundary condition on any (convex) surface patch in three dimensions or curve segment in two dimensions.

Neglecting the error term in (5)-(6), we thus obtain on $\Gamma$

$$
B_{j}^{m}\left[u_{k}\right]=B_{j}^{m}\left[u_{k}+u_{j}\right]=B_{j}^{m}[u], \quad j=1,2, \quad k \neq j .
$$


When $u$ is known on $\Gamma$, equation (8) yields a partial differential equation for the unknown wave field $u_{k}$. In general, it will involve tangential, normal and time derivatives. To restrict $(8)$ to $\Gamma$, we rewrite the normal derivative $\left(\partial_{r_{j}}\right)$ as a combination of radial and tangential $\left(\partial_{r_{k}}\right.$ and $\left.\partial_{\theta_{k}}\right)$ derivatives and then replace radial by time derivatives using (3) or (4). The resulting equation will involve only tangential and time derivatives and thus be restricted to $\Gamma$. Clearly, appropriate initial and boundary conditions must be set on $\Gamma$ for well-posedness. In fact, since the scattered field is initially zero in $\Omega$, both $u_{k}$ and $u_{j}$ vanish on $\Gamma$ at $t=0$.

Remark 2.1. In (8), the absorbing differential operator $B_{j}^{m}$ enables the recovery of every outgoing component of the total field into $\Omega$ in forward time. Instead, we can also consider time-reversed wave fields propagating backwards in time. By replacing the coupled space-time variable $r_{k}-c t$ by $r_{k}+$ ct in the progressive wave expansions (3),(4), we thus obtain differential operators which instead annihilate incoming wave fields.

In the three-dimensional case, for instance, let $B_{j}=B_{j}^{1}$ denote the firstorder Bayliss-Turkel operator:

$$
B_{j}[u]=\left(\frac{1}{c} \frac{\partial}{\partial t}+\frac{\partial}{\partial r_{j}}+\frac{1}{r_{j}}\right) u .
$$

Then, the new operator for the time-reversed total field, $u_{R}$, reads:

$$
B_{R, j}\left[u_{R}\right]=\left(\frac{1}{c} \frac{\partial}{\partial t}-\frac{\partial}{\partial r_{j}}-\frac{1}{r_{j}}\right) u_{R} .
$$

Thus, it is possible to extend wave-splitting even to parts of $\Gamma$, where the forward problem (8) may be ill-posed. To use this time reversed operator, however, we must also set an initial condition for $u_{R, k}$ or $u_{R, j}$ at the final time $t=T$. If $T$ is sufficiently large so that the total wave field has entirely left $\Omega$, we can simply set $u_{R, k}$ and $u_{R, j}$ to zero at time $t=T$ as initial condition for wave splitting in time reversal.

\subsection{One-dimensional wave splitting}

Before proceeding with the higher dimensional case, we briefly consider the much simpler one-dimensional situation to illustrate the basic principle of wave splitting. Hence we consider two sources located inside of two disjoint intervals $S_{1}$ and $S_{2}$, separated by an interval $\Omega_{I}$ - see Fig. 2. Now, as waves 


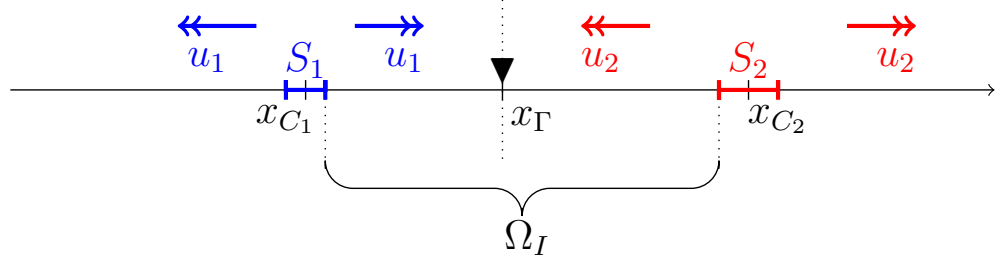

Figure 2: One-dimensional wave splitting. The receiver is located at $x_{\Gamma}$.

are excited inside $S_{1}$ and $S_{2}$, they propagate into $\Omega_{I}$ where they mingle and form the total wave field $u(t, x)$. Since $u$ is initially zero and the wave speed, $c$, is assumed constant outside the two scatterers, the total field consists of a right-moving wave, $u_{1}$, and a left-moving wave, $u_{2}$ :

$$
u(t, x)=u_{1}(x-c t)+u_{2}(x+c t), \quad x \in \Omega_{I}, t \geq 0 .
$$

Next, let $x_{\Gamma}$ denote an observation point located well inside $\Omega_{I}$. Given the time history of the total field $u\left(t, x_{\Gamma}\right)$ at the receiver, we wish to recover the two individual one-way wave fields $u_{1}$ and $u_{2}$.

Following the approach delineated in the previous section for the two- or three-dimensional case, we consider the one-way differential operators

$$
B_{1}[u]:=\frac{\partial u}{\partial t}+c \frac{\partial u}{\partial x}, \quad B_{2}[u]:=\frac{\partial u}{\partial t}-c \frac{\partial u}{\partial x} .
$$

Since $B_{1}\left[u_{1}\right]=B_{2}\left[u_{2}\right]=0$, we thus obtain the one-dimensional counterpart of (8) inside $\Omega_{I}$ :

$$
\begin{aligned}
& B_{1}\left[u_{2}\right]=B_{1}\left[u_{1}\right]+B_{1}\left[u_{2}\right]=B_{1}[u] \\
& B_{2}\left[u_{1}\right]=B_{2}\left[u_{1}\right]+B_{2}\left[u_{2}\right]=B_{2}[u] .
\end{aligned}
$$

Hence, equations (13),(14) correspond to partial differential equations for the unknown wave fields $u_{1}, u_{2}$ which both involve derivatives in space and time. Since $B_{2}\left[u_{2}\right]=0$ inside $\Omega_{I}$, we can replace in (13) the space by the time derivative of $u_{2}$, and similarly for $u_{1}$ in (14):

$$
\begin{aligned}
& B_{1}\left[u_{2}\right]=\frac{\partial u_{2}}{\partial t}+c \frac{\partial u_{2}}{\partial x}=2 \frac{\partial u_{2}}{\partial t} \\
& B_{2}\left[u_{1}\right]=\frac{\partial u_{1}}{\partial t}-c \frac{\partial u_{1}}{\partial x}=2 \frac{\partial u_{1}}{\partial t} .
\end{aligned}
$$




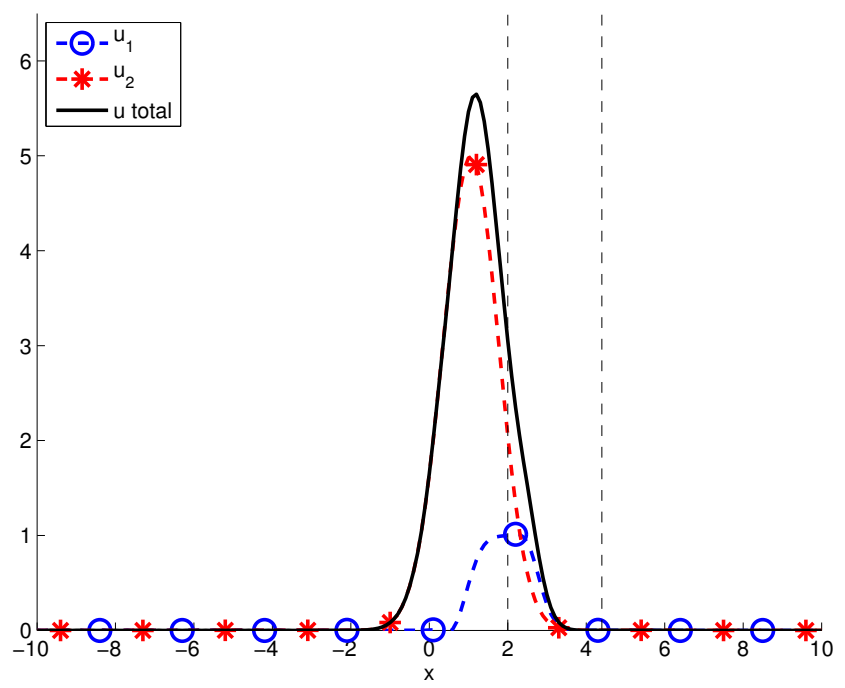

Figure 3: One-dimensional wave splitting. Snapshot of the total field $u$ with the rightand left-propagating waves $u_{1}$ and $u_{2}$ at time $t=7.45$. The two receivers are located at $x_{\Gamma}=2$ and $x_{\Gamma}=4.4$ (vertical dashed lines).

Setting $x=x_{\Gamma}$ in the resulting equations, we obtain the two ordinary equations:

$$
\begin{aligned}
\frac{\partial u_{2}}{\partial t}\left(x_{\Gamma}+c t\right) & =\frac{1}{2} B_{1}[u]\left(t, x_{\Gamma}\right), \\
\frac{\partial u_{1}}{\partial t}\left(x_{\Gamma}-c t\right) & =\frac{1}{2} B_{2}[u]\left(t, x_{\Gamma}\right) .
\end{aligned}
$$

To recover $u_{1}$ or $u_{2}$, we shall integrate (15) and (16) in time, together with homogeneous initial conditions, since the signal at $x_{\Gamma}$ is initially zero. Here, we opt for the Crank-Nicolson scheme and let $u_{2}^{n} \simeq u_{2}\left(x_{\Gamma}+c t^{n}\right)$, $u_{1}^{n} \simeq u_{1}\left(x_{\Gamma}-c t^{n}\right)$ denote the numerical approximations at time $t^{n}=n \Delta t$, $\Delta t>0$. The discrete time-marching scheme then reads:

$$
\begin{array}{ll}
u_{2}^{n+1}=u_{2}^{n}+\frac{\Delta t}{4}\left(B_{1}[u]\left(t^{n+1}, x_{\Gamma}\right)+B_{1}[u]\left(t^{n}, x_{\Gamma}\right)\right), \quad n \geq 0, \\
u_{1}^{n+1}=u_{1}^{n}+\frac{\Delta t}{4}\left(B_{2}[u]\left(t^{n+1}, x_{\Gamma}\right)+B_{2}[u]\left(t^{n}, x_{\Gamma}\right)\right), \quad n \geq 0,
\end{array}
$$

and $u_{2}^{0}=0, u_{1}^{0}=0$. The right-hand-side is discretized using centered finite 

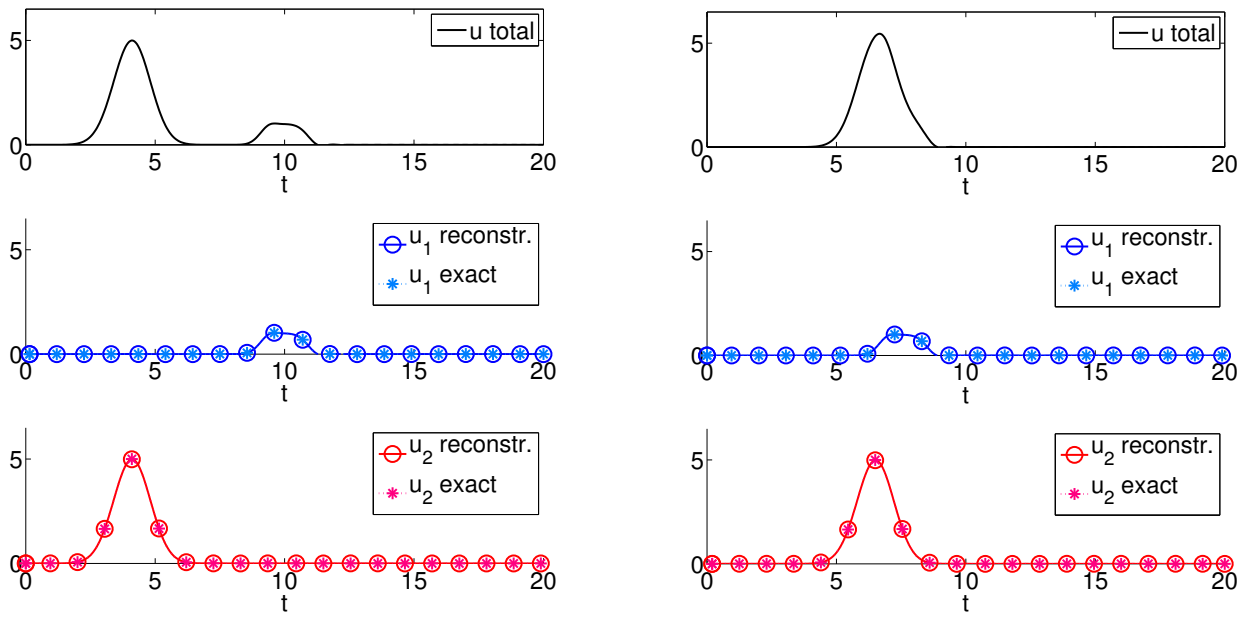

Figure 4: One-dimensional wave splitting. Reconstruction of the time history of $u_{1}$ and $u_{2}$ at $x_{\Gamma}=4.4$ (left column) and $x_{\Gamma}=2$ (right column) from the total field $u$ using (18) and (17).

differences in time and in space in our simulation, assuming we know $u$ in a small neighborhood of $x_{\Gamma}$.

For illustration, we consider a numerical experiment with $c=1$ and the two intervals $S_{1}=[-8,-3], S_{2}=[5,12]$ centered about $x_{C_{1}}=-5.5$, $x_{C_{2}}=8.5$, respectively. Inside each interval, we initialize a Gaussian-like wave

$$
u_{1}(x, t)=e^{-\left(\left(x-x_{C_{1}}\right)-c t\right)^{4}} \chi_{S_{1}}(x), \quad u_{2}(x, t)=5 e^{-\left(\left(x-x_{C_{2}}\right)+c t\right)^{2}} \chi_{S_{2}}(x),
$$

where $\chi_{S}$ denotes the characteristic function of $S$. Hence the total field,

$$
u(x, t)=e^{-\left(\left(x-x_{C_{1}}\right)-c t\right)^{4}} \chi_{S_{1}}(x)+5 e^{-\left(\left(x-x_{C_{2}}\right)+c t\right)^{2}} \chi_{S_{2}}(x),
$$

is identically zero at $t=0$ inside $\Omega_{I}$.

In Fig. 3, we display a snapshot of the total field $u(t, x)$ at time $t=7.45$ together with the right- and left-propagating waves $u_{1}$ and $u_{2}$ that we wish to recover. For the reconstruction of $u_{1}$ and $u_{2}$, we consider two distinct locations: $x_{\Gamma}=2$ and $x_{\Gamma}=4.4$. At $x_{\Gamma}=4.4, u_{1}$ and $u_{2}$ can easily be separated because of their very different arrival times - see left column of Fig. 4. At $x_{\Gamma}=2$, however, the two fields are completely superimposed and thus difficult to separate by eye-ball only - see right column of Fig. 4. In either 
case, our approach allows us to recover the full time history of $u_{1}$ and $u_{2}$ at the receiver's location with high accuracy.

For simplicity, here the considered wave speed $c$ is constant everywhere; hence, the exact wave fields $u_{1}, u_{2}$ and $u$ are readily available. Our wave splitting approach, however, also immediately applies to the situation when $c(x)$ varies inside $S_{1}$ and $S_{2}$.

\section{Two-dimensional case}

We shall now apply the wave splitting technique delineated in Section 2 to a typical two-dimensional configuration. Hence, we assume that the total field $u$ is recorded on a circle $\Gamma$ centered at either of the two sources $C_{1}$ or $C_{2}$. Given the measured values of $u$ at times $0 \leq t \leq T$ on $\Gamma$, here a circle centered about $C_{1}$ - see Fig. 5 -, we shall recover both outgoing fields $u_{1}$ and $u_{2}$ using first-order absorbing differential operators on the time interval $[0, T]$.

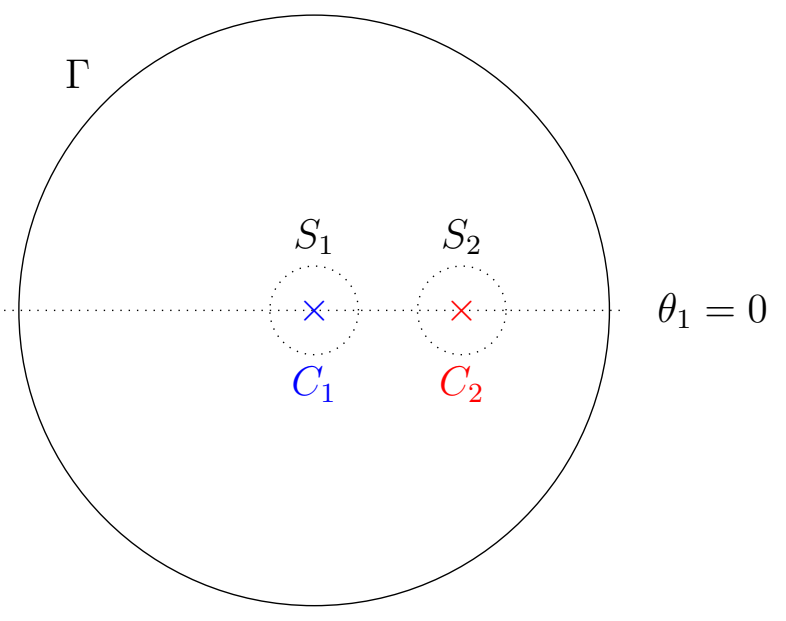

Figure 5: The two sources are located inside $S_{1}$ and $S_{2}$, while the measurements are located on the circle $\Gamma$, centered at $C_{1}$.

\subsection{Recovery of $u_{1}$}

First, we shall show how to recover $u_{1}$ on $\Gamma$ from $u$. Let $\left(r_{k}, \theta_{k}\right)$ denote polar coordinates centered at $C_{k}, k=1,2$. Now, let the $\theta_{1}=0$ axis coincide with the line segment connecting $C_{1}$ and $C_{2}$, which is always possible by rotating the coordinate system. Following the derivation in Section 2, we 
apply $B_{2}:=B_{2}^{1}$ from (6) to the measured total field $u$, which yields the two-dimensional counterpart of (8):

$$
\left(\frac{1}{c} \frac{\partial}{\partial t}+\frac{\partial}{\partial r_{2}}+\frac{1}{2 r_{2}}\right) u_{1}=\left(\frac{1}{c} \frac{\partial}{\partial t}+\frac{\partial}{\partial r_{2}}+\frac{1}{2 r_{2}}\right) u
$$

To restrict equation (19) for $u_{1}$ to $\Gamma$, we first change coordinates and then replace any remaining normal by time derivatives using that $B_{1}\left[u_{1}\right]=0$. More specifically, we use

$$
\begin{aligned}
\frac{\partial}{\partial r_{2}} & =\frac{r_{2}+\ell \cos \left(\theta_{2}\right)}{r_{1}} \frac{\partial}{\partial r_{1}}+\frac{\ell \sin \left(\theta_{2}\right)}{r_{1}^{2}} \frac{\partial}{\partial \theta_{1}} \\
\frac{\partial}{\partial r_{1}}\left(\sqrt{r_{1}} u_{1}\right) & =-\frac{1}{c} \frac{\partial}{\partial t}\left(\sqrt{r_{1}} u_{1}\right)
\end{aligned}
$$

with $\ell$ the distance between $C_{1}$ and $C_{2}$, to rewrite (19) for the leading order term $f_{1}:=f_{1,0}\left(r_{1}-c t, \theta_{1}\right)$ of the series expansion in (4) as

$$
\left(\alpha_{1}\left(\theta_{1}\right) \frac{\partial}{\partial t}+\beta_{1}\left(\theta_{1}\right) \frac{\partial}{\partial \theta_{1}}+\gamma_{1}\left(\theta_{1}\right)\right) f_{1}=\left(\frac{1}{c} \frac{\partial}{\partial t}+\frac{\partial}{\partial r_{2}}+\frac{1}{2 r_{2}}\right) u
$$

Here, the coefficient functions $\alpha_{1}, \beta_{1}$ and $\gamma_{1}$ in (22) are given by

$$
\begin{aligned}
\alpha_{1}\left(\theta_{1}\right) & =\frac{\sqrt{r_{1}^{2}+\ell^{2}-2 r_{1} \ell \cos \left(\theta_{1}\right)}-r_{1}+\ell \cos \left(\theta_{1}\right)}{c \sqrt{r_{1}} \sqrt{r_{1}^{2}+\ell^{2}-2 r_{1} \ell \cos \left(\theta_{1}\right)}}, \\
\beta_{1}\left(\theta_{1}\right) & =\frac{\ell \sin \left(\theta_{1}\right)}{r_{1} \sqrt{r_{1}} \sqrt{r_{1}^{2}+\ell^{2}-2 r_{1} \ell \cos \left(\theta_{1}\right)}}, \\
\gamma_{1}\left(\theta_{1}\right) & =\frac{\ell \cos \left(\theta_{1}\right)}{2 r_{1} \sqrt{r_{1}} \sqrt{r_{1}^{2}+\ell^{2}-2 r_{1} \ell \cos \left(\theta_{1}\right)}} .
\end{aligned}
$$

Note that both $\ell$ and $r_{1}$ are constant on $\Gamma$, while

$$
r_{1}^{2}+\ell^{2}-2 r_{1} \ell \cos \left(\theta_{1}\right)>0, \quad \forall \theta_{1} \in[-\pi, \pi] .
$$

In (22), we have derived a partial differential equation for $f_{1}\left(r_{1}-c t, \theta_{1}\right)$, which only involves time and space derivatives of $f_{1}$ tangential to $\Gamma$. To determine the domain of validity and the well-posedness of the hyperbolic equation (22), we now study the sign of each coefficient, which determines the 


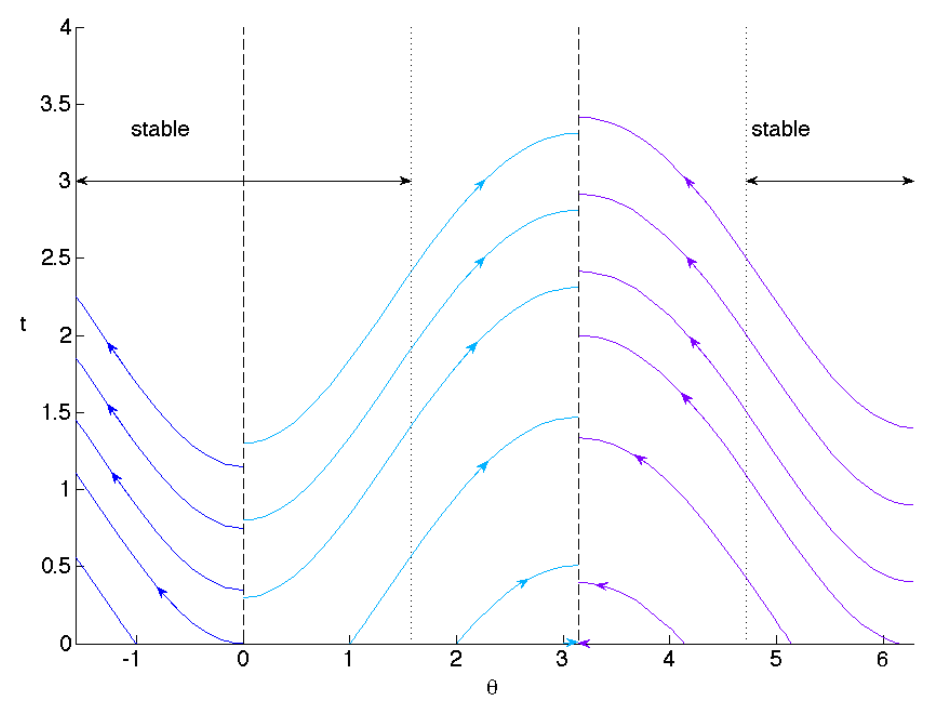

Figure 6: Characteristic curves for (22) starting from different angles $\theta$

directions of the characteristics and thus dictates the appropriate boundary conditions, if any, needed. As initial condition, we set $u_{1}$ to zero on $\Gamma$, as $u$ is initially zero inside $\Omega$.

Since

$$
r_{1}^{2}+\ell^{2}-2 r_{1} \ell \cos \left(\theta_{1}\right) \geq r_{1}^{2}+\ell^{2} \cos \left(\theta_{1}\right)^{2}-2 r_{1} \ell \cos \left(\theta_{1}\right)=\left(r_{1}-\ell \cos \left(\theta_{1}\right)\right)^{2},
$$

we have

$$
\sqrt{r_{1}^{2}+\ell^{2}-2 r_{1} \ell \cos \left(\theta_{1}\right)} \geq r_{1}-\ell \cos \left(\theta_{1}\right) .
$$

Therefore, $\alpha_{1}\left(\theta_{1}\right) \geq 0$ for all $\theta_{1} \in[-\pi, \pi]$; note that $\alpha_{1}=0$ for $\theta_{1}=0, \pi$.

We now proceed likewise for $\beta_{1}$ and $\gamma_{1}$. Since $\ell$ and $r_{1}$ are positive, $\beta_{1}$ is nonnegative for $\theta_{1} \in[0, \pi]$. For $\theta_{1}=0, \pi, \beta_{1}\left(\theta_{1}\right)$ vanishes and hence (22) reduces to a trivial equation (without derivatives) in time. In that sense, both $\theta_{1}=0, \pi$ are singular points. From (25), we infer that $\gamma_{1}$ is positive for $\theta_{1} \in(-\pi / 2, \pi / 2)$, negative for $\theta_{1} \in(\pi / 2,3 \pi / 2)$, and vanishes for $\theta_{1}=-\pi / 2, \pi / 2$. Since (22) is unstable when the signs of $\gamma_{1}$ and $\alpha_{1}$ do not match, and $\alpha_{1}\left(\theta_{1}\right) \geq 0$ for all $\theta_{1}$, we conclude that (22) is stable only for $\theta_{1} \in[-\pi / 2, \pi / 2]-$ see Fig. 8 .

In Fig. 6, we display the characteristics of (22) and clearly observe two distinguished points: $\theta_{1}=0$ and $\theta_{1}=\pi$. At $\theta_{1}=0$, the characteristics are 


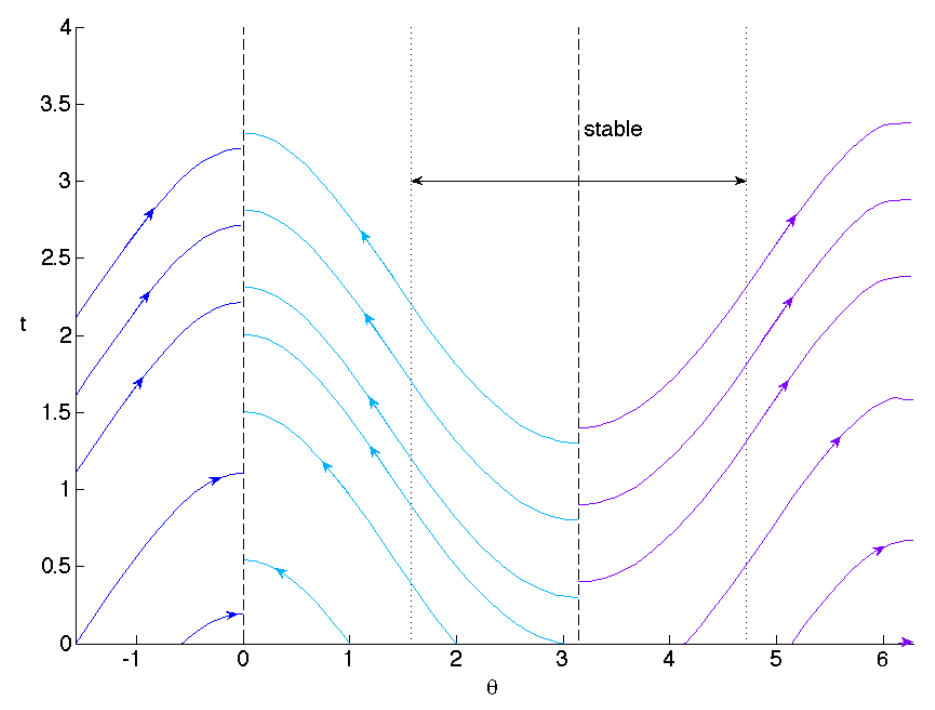

Figure 7: Characteristic curves for (27) starting from different angles $\theta$

always incoming and thus $\theta_{1}=0$ yields an appropriate initial condition to recover the signal in both intervals $[0, \pi)$ and $(-\pi, 0]$. On the other hand, $\theta_{1}=\pi$ is never reached by any characteristic (except for that starting from $\theta=\pi$ itself). From these considerations, we impose at $\theta_{1}=0$ a Dirichlet boundary condition to solve (22) in a stable manner on the two independent quarter-circles $[0, \pi / 2]$ and $[-\pi / 2,0]$.

To determine $u_{1}$ on the remaining half-circle, $\theta_{1} \in[\pi / 2,3 \pi / 2]$, where $u_{1}$ is exponentially growing, we consider the time-reversed counterpart of (22). Hence, we let $u_{R}$ denote the time-reversed total wave field and apply the time-reversed first-order Bayliss-Turkel operator $B_{R, 2}$ - see Remark 2.1 which yields:

$$
\left(\frac{1}{c} \frac{\partial}{\partial t}-\frac{\partial}{\partial r_{2}}-\frac{1}{2 r_{2}}\right) u_{R, 1}=\left(\frac{1}{c} \frac{\partial}{\partial t}-\frac{\partial}{\partial r_{2}}-\frac{1}{2 r_{2}}\right) u_{R}
$$

Following the previous derivation (19)-(22), we replace the normal derivative by tangential and time derivatives in (26) using (20), (21). This yields the time-reversed counterpart of $(22)$ for the leading term, $f_{R, 1}$, in the series 
expansion of the time-reversed wave field:

$$
\left(\alpha_{R, 1}\left(\theta_{1}\right) \frac{\partial}{\partial t}+\beta_{R, 1}\left(\theta_{1}\right) \frac{\partial}{\partial \theta_{1}}+\gamma_{R, 1}\left(\theta_{1}\right)\right) f_{R, 1}=\left(\frac{1}{c} \frac{\partial}{\partial t}-\frac{\partial}{\partial r_{2}}-\frac{1}{2 r_{2}}\right) u_{R}
$$

Here, the coefficient functions are given by

$$
\alpha_{R, 1}\left(\theta_{1}\right)=\alpha_{1}\left(\theta_{1}\right), \quad \beta_{R, 1}\left(\theta_{1}\right)=-\beta_{1}\left(\theta_{1}\right), \quad \gamma_{R, 1}\left(\theta_{1}\right)=-\gamma_{1}\left(\theta_{1}\right),
$$

with $\alpha_{1}, \beta_{1}$ and $\gamma_{1}$ as in (23)-(25). Again, $\alpha_{R, 1}$ and $\beta_{R, 1}$ are zero at $\theta_{1}=0, \pi$, while $\alpha_{R, 1}$ is always positive. The sign of $\beta_{R, 1}$ is now opposite to that of $\beta_{1}$, as confirmed by the direction of the corresponding characteristics - see Fig. 7 . Moreover, since $\gamma_{R, 1}$ and $\gamma_{1}$ have also opposite signs, equation (27) is now stable on $[\pi / 2,3 \pi / 2]$, where both $\alpha_{R, 1}$ and $\gamma_{R, 1}$ are nonnegative. According to the direction of the characteristics, we must now set the boundary condition at $\theta_{1}=\pi$.

The initial condition for the time reversed equation (27) is given by the value of $u$ at $t=T$, where $T$ is the final recording or simulation time. Since it is no longer possible to distinguish $u_{1}$ from $u_{2}$ at later times, we must set the final time $T$ sufficiently large so that the total wave field has left the computational domain and thus vanishes throughout $\Omega$. Clearly, the true scattered field generally never vanishes completely but only decays rapidly. In practice, we stop the forward computation and declare the remaining wave field to be zero, once it is smaller than $5 \%$ of the total wave field. Hence, we set $f_{1}=0$ at $t=T$, or $f_{R, 1}=0$ at $t=0$, equivalently.

Finally, we recover $u_{1}$ by solving $(22)$ on $[-\pi / 2,0[\cup] 0, \pi / 2]$ forward in time and (27) backward in time on $[\pi / 2, \pi[\cup] \pi, 3 \pi / 2]$, as shown in Fig. 8. In pratice, due to the two dividing characteristics at $\theta_{1}=0, \pi$, we actually solve four partial differential equations on four quarter-circles independently of each other. Since (22) and (27) both involve only partial derivatives but no integrals, our approach is local in time and in space.

In fact, to reconstruct the signal at any fixed point $P$ at some time $t_{0}>0$, we need to know the total field only on the fraction of the circle from $\theta_{1}=0$ to $P$, or from $\theta_{1}=\pi$ to $P$, respectively, and for $t \in\left[0, t_{0}\right]$. Thus it is even possible to recover the scattered field components $u_{1}$ and $u_{2}$ only over a part of the boundary $\Gamma$, as long as $u$ is known there too.

Note that in the special case when $u_{1}$ or $u_{2}$ corresponds to a purely radial wave generated by a point source, the corresponding partial differential equation (22) or (27) reduces to an ordinary differential equation, which can be solved for any particular angle independently, regardless of the shape of $\Omega$. 


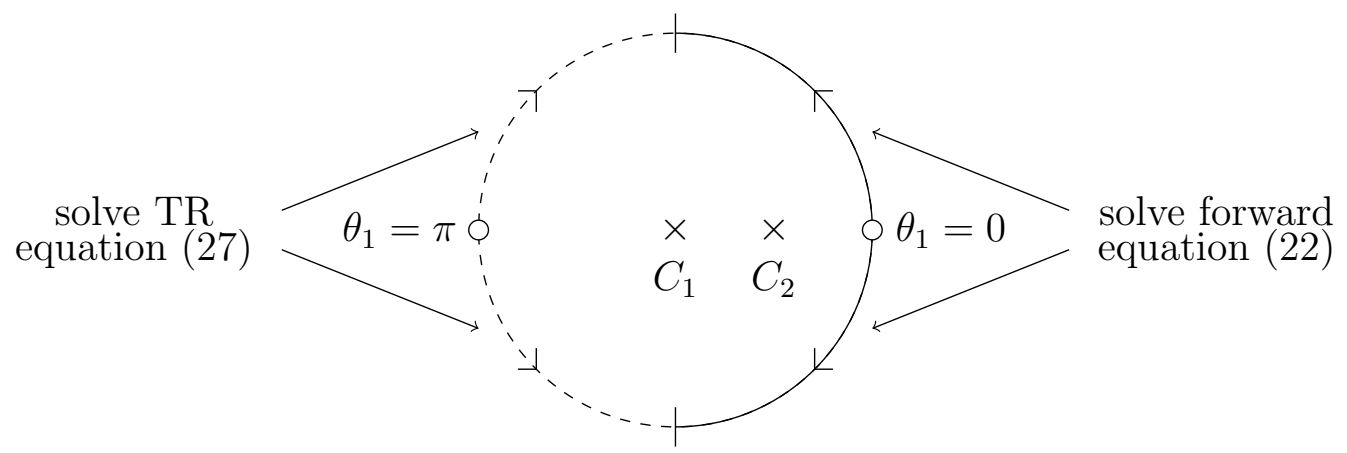

Figure 8: The domain of validity for the recovery of $f_{1}$ and $f_{R, 1}$. Plain line: solve the forward equation (22). Dashed line: solve the time-reversed (TR) equation (27). The characteristics point away from the two small circles at $\theta_{1}=0, \pi$, where the boundary conditions are set, towards the vertical bars at $\theta_{1}=-\pi / 2, \pi / 2$, which mark the end of validity of each quarter-circle.

\subsection{Recovery of $u_{2}$}

In the previous section, we derived equations (22) and (27) to recover $u_{1}$ on the entire circle $\Gamma$. From the recorded data $u$, we can thus simply recover $u_{2}$ by subtraction. However, we can also recover $u_{2}$ from a new independent equation by applying $B_{1}:=B_{1}^{1}$ from (6) instead of $B_{2}$ to $u$ :

$$
\left(\frac{1}{c} \frac{\partial}{\partial t}+\frac{\partial}{\partial r_{1}}+\frac{1}{2 r_{1}}\right) u_{2}=\left(\frac{1}{c} \frac{\partial}{\partial t}+\frac{\partial}{\partial r_{1}}+\frac{1}{2 r_{1}}\right) u \text {. }
$$

Again, we use (20) to replace the radial derivative on the left of (29) by a combination of radial and tangential derivatives. Next, we use (7) with $m=1$ and $k=2$, that is

$$
\frac{\partial}{\partial r_{2}}\left(\sqrt{r_{2}} u_{2}\right)=-\frac{1}{c} \frac{\partial}{\partial t}\left(\sqrt{r_{2}} u_{2}\right)
$$

to replace the remaining radial derivative by a time derivative. Thus, we obtain the following partial differential equation for the leading order term $f_{2}:=f_{2,0}\left(r_{2}-c t, \theta_{2}\right)$ in the series expansion (4) of $u_{2}$ :

$$
\left(\alpha_{2}\left(\theta_{1}\right) \frac{\partial}{\partial t}+\beta_{2}\left(\theta_{1}\right) \frac{\partial}{\partial \theta_{1}}+\gamma_{2}\left(\theta_{1}\right)\right) f_{2}=\left(\frac{1}{c} \frac{\partial}{\partial t}+\frac{\partial}{\partial r_{1}}+\frac{1}{2 r_{1}}\right) u
$$

Here, the coefficient functions are given by 


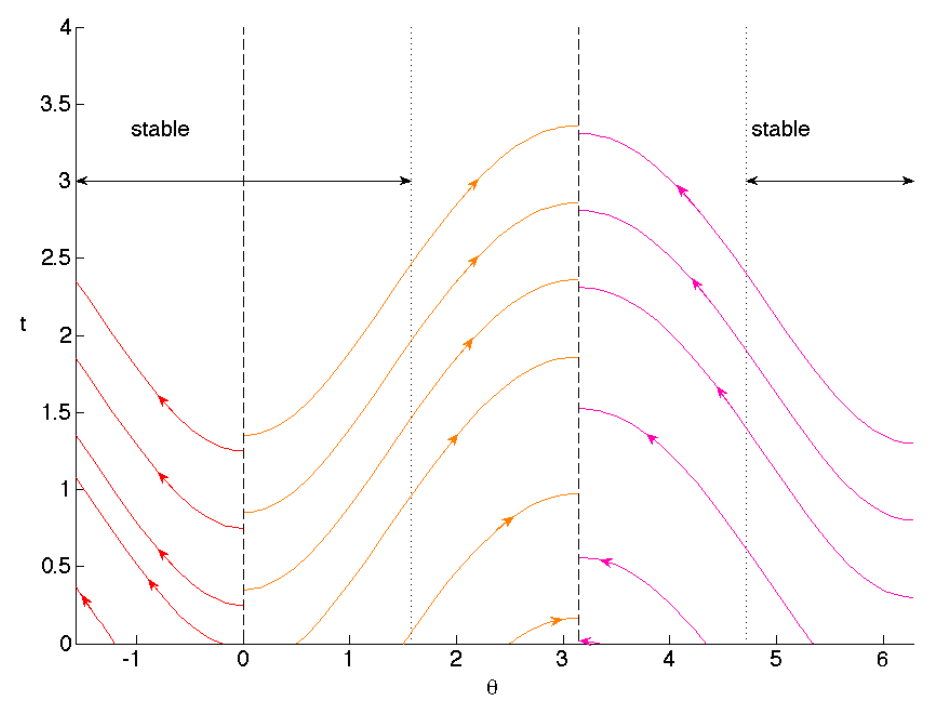

Figure 9: Characteristic curves for (31) starting from different angles $\theta$

$$
\begin{aligned}
\alpha_{2}\left(\theta_{1}\right) & =\frac{\sqrt{r_{1}^{2}+\ell^{2}-2 r_{1} \ell \cos \left(\theta_{1}\right)}-r_{1}+\ell \cos \left(\theta_{1}\right)}{c \sqrt[4]{r_{1}^{2}+\ell^{2}-2 r_{1} \ell \cos \left(\theta_{1}\right)}\left(r_{1}-\ell \cos \left(\theta_{1}\right)\right)} \\
\beta_{2}\left(\theta_{1}\right) & =\frac{\ell \sin \left(\theta_{1}\right)}{r_{1} \sqrt[4]{r_{1}^{2}+\ell^{2}-2 r_{1} \ell \cos \left(\theta_{1}\right)}\left(r_{1}-\ell \cos \left(\theta_{1}\right)\right)}, \\
\gamma_{2}\left(\theta_{1}\right) & =\frac{1}{2 \sqrt[4]{r_{1}^{2}+\ell^{2}-2 r_{1} \ell \cos \left(\theta_{1}\right)}\left(r_{1}-\ell \cos \left(\theta_{1}\right)\right)}\left(\frac{\ell \cos \left(\theta_{1}\right)}{r_{1}}-\frac{\ell^{2} \sin \left(\theta_{1}\right)^{2}}{r_{1}^{2}+\ell^{2}-2 r_{1} \ell \cos \left(\theta_{1}\right)}\right) .
\end{aligned}
$$

Note that $\alpha_{2}, \beta_{2}$ and $\gamma_{2}$ are still functions of $\theta_{1}$, since we recover $u_{2}$ on the circle $\Gamma$ still centered at $C_{1}$. Remarkably, the signs of $\alpha_{2}, \beta_{2}$ and $\gamma_{2}$ coincide with those of $\alpha_{1}, \beta_{1}$ and $\gamma_{1}$, respectively, and so do the characteristics curves - see Fig. 9. Therefore, the same conclusions hold about the initial condition, the boundary conditions at $\theta_{1}=0, \pi$, and the stability properties of $(31)$ on $[-\pi / 2,0) \cup(0, \pi / 2]$. Similarly, we use the time reversed counterpart of (31), which is omitted here, to reconstructed $u_{2}$ on $[\pi / 2, \pi) \cup(\pi, 3 \pi / 2]-$ see Fig. 8.

\subsection{Numerical discretization}

To determine $u_{1}$ on $\Gamma$, we shall solve (22) or (27) numerically, depending on the quarter-circle considered. For the numerical discretization, we opt for the standard Crank-Nicolson scheme in time and upwinding finite differences 
in space following the characteristics. The numerical discretization of (27) on $\left[\frac{\pi}{2}, \pi\right)$, for instance, reads:

$$
\begin{gathered}
\alpha(i \Delta \theta) \frac{f_{i}^{n+1}-f_{i}^{n}}{\Delta t}+\frac{1}{2} \beta(i \Delta \theta)\left(\frac{f_{i+1}^{n+1}-f_{i}^{n+1}}{\Delta \theta}+\frac{f_{i+1}^{n}-f_{i}^{n}}{\Delta \theta}\right) \\
+\frac{1}{2} \gamma(i \Delta \theta)\left(f_{i}^{n+1}+f_{i}^{n}\right)=\frac{1}{2}\left(g_{i}^{n+1}+g_{i}^{n}\right),
\end{gathered}
$$

with $g_{i}^{n}=B_{R, 2}[u]\left(t^{n}, i \Delta \theta\right)$ and $f_{1}^{0} \equiv 0$ at $t=0$.

To compute the right-hand-side $g_{i}^{n}=B_{R, 2}[u]\left(t^{n}, i \Delta \theta\right)$ in (27), the wave field $u$ and its radial derivative are both computed with the finite element software FreeFem $++[21]$. Indeed, from the variational formulation, we easily get an accurate approximation of the radial derivative at $\Gamma$, since

$$
\int_{\Gamma} \frac{\partial u}{\partial r_{j}} v=\int_{\Gamma} \frac{\partial u}{\partial n} v=\iint_{\Omega} \frac{\partial^{2} u}{\partial t^{2}} w+\iint_{\Omega} \nabla u \cdot \nabla w .
$$

Here, $\Gamma$ denotes a circle centered at $C_{j}$, while $w \in H^{1}(\Omega)$ is an extension of the trace $v \in H^{\frac{1}{2}}(\Gamma)$ into $\Omega$, which vanishes on all vertices inside $\Omega$. For the time and tangential derivatives, we use finite differences and cubic spline interpolation. As the numerical discretizations used on the left and right side of (32) differ, we also avoid any potential "inverse crime".

\section{Numerical examples}

To illustrate the usefulness of the wave splitting approach described in Section 3, we now consider numerical experiments in two different geometric configurations.

\subsection{Two point sources}

First, we consider two point sources $F_{1}(t)$ and $F_{2}(t)$, located at $C_{1}$ and $C_{2}$ - see Fig. 1. Each source generates an outgoing wave field $u_{j}$. At the outer boundary, we record their sum: $u=u_{1}+u_{2}$. Given that observed data on $\Gamma$, we wish to recover the two separate components $u_{1}$ and $u_{2}$. Hence, $u$ satifies the wave equation

$$
\frac{\partial^{2} u}{\partial t^{2}}-c^{2} \Delta u=F_{1}(t) \delta_{x=C_{1}}+F_{2}(t) \delta_{x=C_{2}}, \quad \text { in } \mathbb{R}^{2},
$$

with homogeneous initial conditions. The time dependence of the two sources $F_{1}$ and $F_{2}$, shown in Fig. 10, is given by 

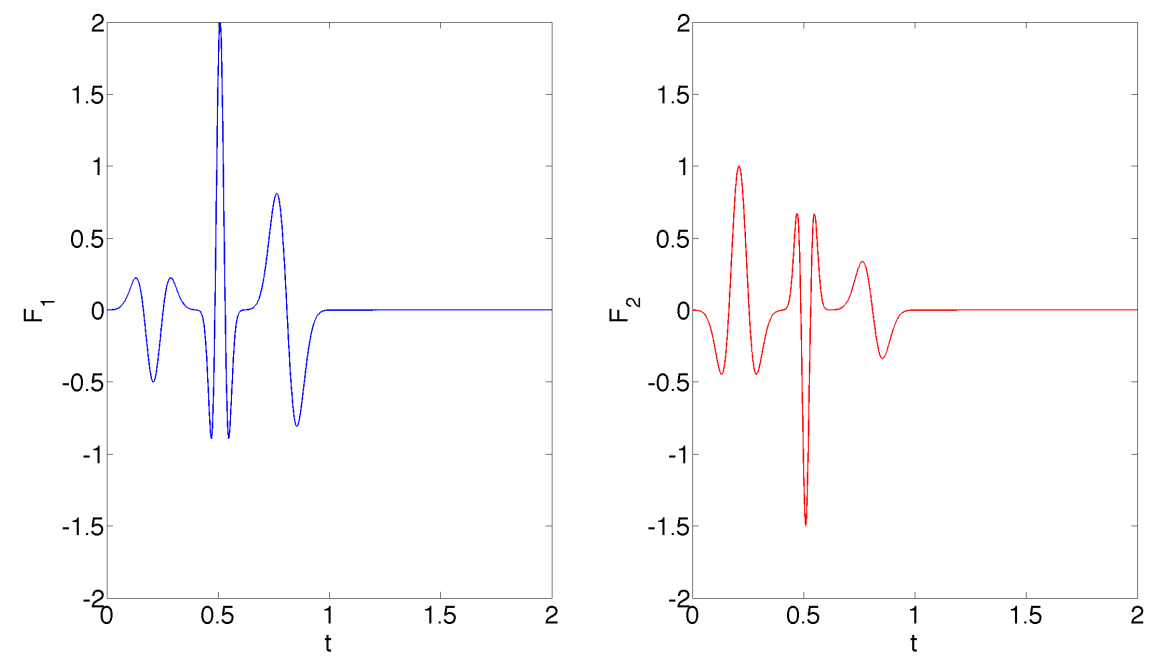

Figure 10: Time dependence of the two point sources $F_{1}$ and $F_{2}$ in (34).

$$
\begin{aligned}
F_{1}(t)= & {\left[1-2 \pi^{2}\left(\nu\left(t-d_{1}\right)-1\right)^{2}\right] e^{-\pi^{2}\left(\nu\left(t-d_{1}\right)-1\right)^{2}} } \\
& -\frac{3}{2}\left[1-2 \pi^{2}\left(2 \nu\left(t-d_{2}\right)-1\right)^{2}\right] e^{-\pi^{2}\left(2 \nu\left(t-d_{2}\right)-1\right)^{2}} \\
& -\frac{3}{5} \pi^{2}\left(\nu\left(t-d_{3}\right)-1\right) e^{-\pi^{2}\left(\nu\left(t-d_{3}\right)-1\right)^{2}} \\
F_{2}(t)= & -\frac{1}{2}\left[1-2 \pi^{2}\left(\nu\left(t-d_{1}\right)-1\right)^{2}\right] e^{-\pi^{2}\left(\nu\left(t-d_{1}\right)-1\right)^{2}} \\
& +2\left[1-2 \pi^{2}\left(2 \nu\left(t-d_{2}\right)-1\right)^{2}\right] e^{-\pi^{2}\left(2 \nu\left(t-d_{2}\right)-1\right)^{2}} \\
& -\frac{1}{4} \pi^{2}\left(\nu\left(t-d_{3}\right)-1\right) e^{-\pi^{2}\left(\nu\left(t-d_{3}\right)-1\right)^{2}}
\end{aligned}
$$

with $d_{1}=0.01, d_{2}=0.41, d_{3}=0.61$ and $\nu=5$. They consist of the superposition of three time shifted Ricker-like sources. Next, we set $c \equiv 1$, the wavelength $\lambda=0.2$ and let $C_{1}$ and $C_{2}$ equal $(-\lambda / 2,0)$ and $(\lambda / 2,0)$, respectively. The total field is recorded on a circle $\Gamma$ centered at $C_{1}$ with radius $5 \lambda / 2$ - see Fig. 11.

In Fig. 12, we display the time history of the recovered two wave fields $u_{1}$ and $u_{2}$ at $P_{1}$, located on $\Gamma$ at $\theta_{1}=-\pi / 4$. Due to the particularly simple set-up, here both $u_{1}$ and $u_{2}$ are known explicitly and for all time. In the top left frame, we compare $u_{1}$, obtained by solving (22), with the exact wave field and observe that both coincide quite well. Similarly, the wave field $u_{2}$, recovered by solving (31), compares favorably with the exact wave field emanating from $C_{2}$. Then in the top right frame, we compare the sum $u_{1}+u_{2}$ of 


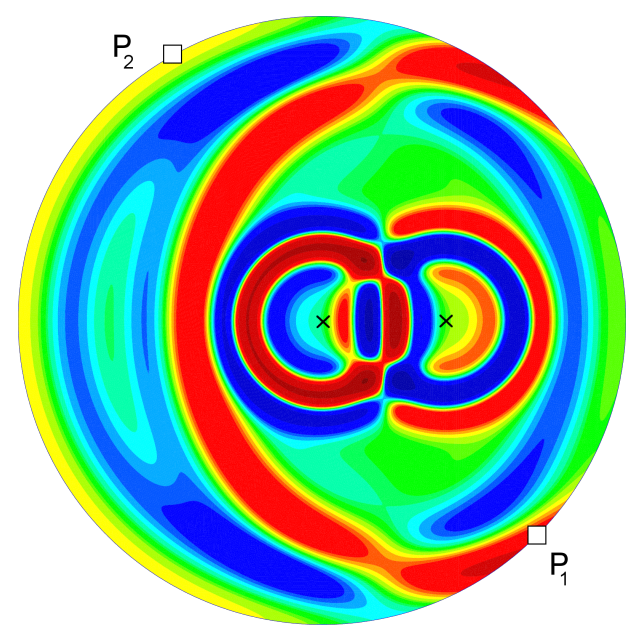

Figure 11: Two point sources. Snapshot of the total wave field at $t=0.64$.

the two recovered fields from (22) and (31), with the recorded total field $u$. Here we observe tiny errors in the reconstruction due to the first-order approximation in the progressive wave expansion (4). Nevertheless, when we add the recovered signals $u_{1}$ and $u_{2}$ and compare them to the recorded total field, the discrepancy is smaller than $1 \%$ of the average amplitude.

In Fig. 13, we show the time history of the recovered fields $u_{1}$ and $u_{2}$ at $P_{2}$ located at $\theta_{1}=3 \pi / 5$. To recover $u_{1}$, we thus solve the time reversed equation (27), and the time reversed counterpart of (31) to obtain $u_{2}$. Again, the recovered outgoing wave fields $u_{1}$ and $u_{2}$ agree remarkably well with the exact wave fields.

\subsection{Scattering from a sound-soft inclusion}

Next, we consider scattering from a sound-soft inclusion. Here, the total field consists of the incident wave $u^{I}$ and the scattered wave $u^{S}$. The incident field, generated by the same point source $F_{1}$ as in (35), impinges upon a sound-soft fish-shaped obstacle, shown in Fig. 14. It is then scattered into the surrounding medium, where it mingles with the incident field as it reaches the outer circle $\Gamma$.

Again, we set $\nu=5, c=1$ and $\lambda=0.2$. The point source, however, is located at $C_{1}=(-3 \lambda / 4,0)$ such that the distance between the source and the head of the fish (first impact) is $\lambda$. In contrast to the previous case, the scattered field $u_{2}=u^{S}$ is neither purely radial nor explicitly known. Whereas $C_{1}$ corresponds precisely to the source location, we set $C_{2}$ to $(\lambda / 2,0)$, 

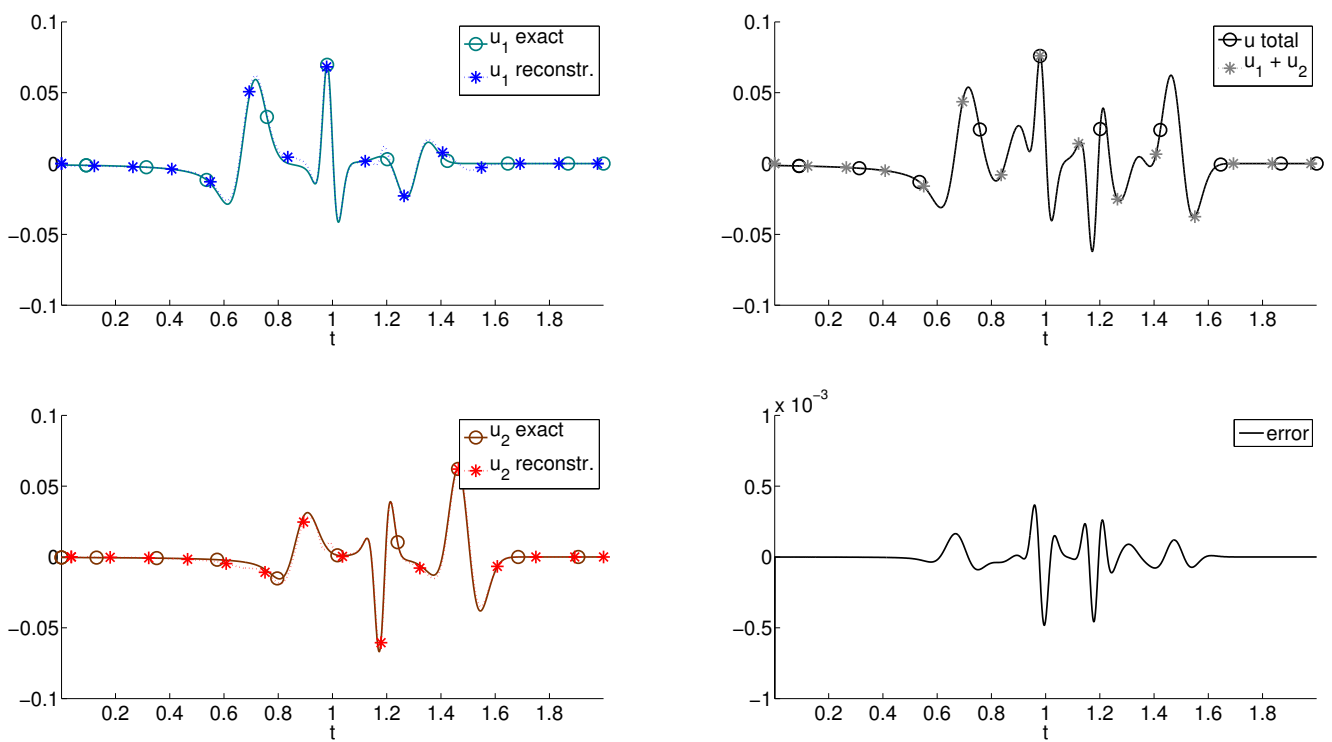

Figure 12: Two point sources. Both $u_{1}$ and $u_{2}$, recovered from total field measurements $u$, are shown at location $P_{1}$ vs. time. Top-left: recovery of $u_{1}$ by solving (22). Bottomleft: recovery of $u_{2}$ by solving (31). Top-right: total field $u$. Bottom-right: discrepancy between $u$ and the sum of the recovered fields $u_{1}+u_{2}$.
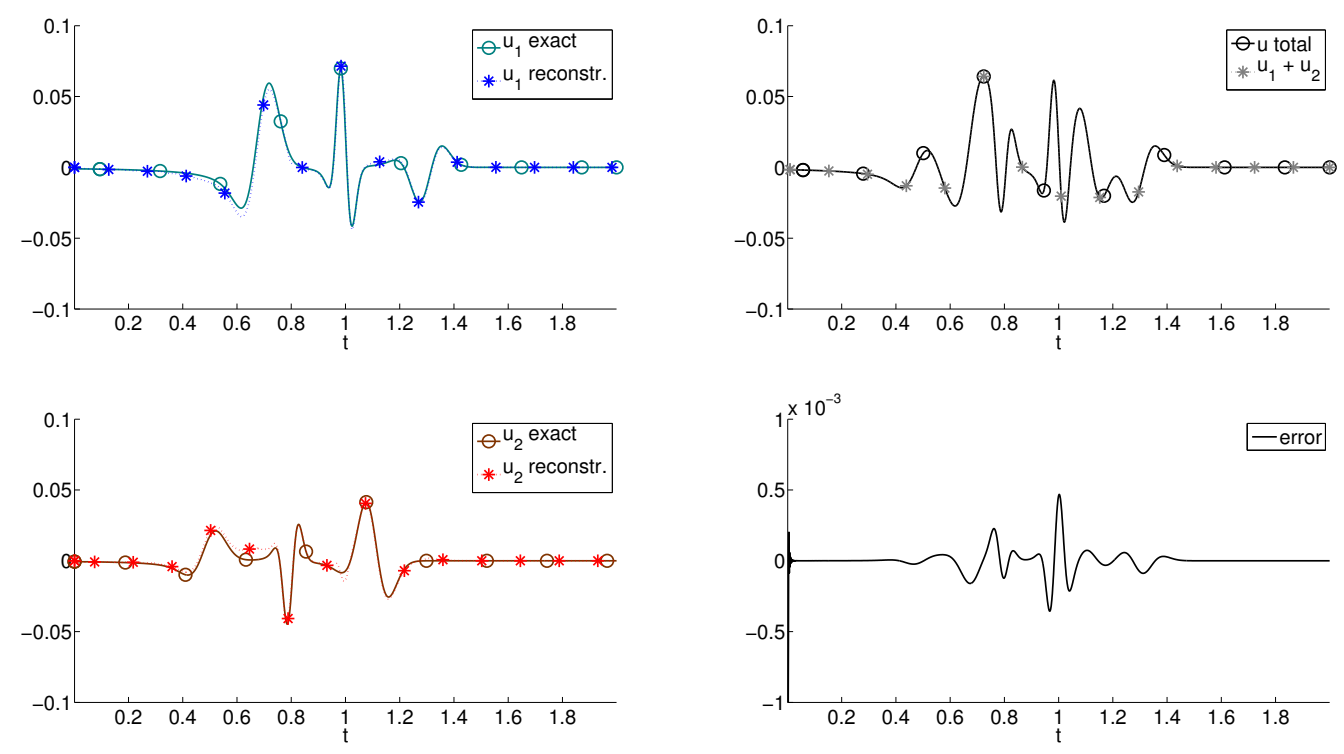

Figure 13: Two point sources. Both $u_{1}$ and $u_{2}$, recovered from total field measurements $u$, are shown at location $P_{2}$ vs. time. Top-left: recovery of $u_{1}$ by solving (27). Bottom-left: recovery of $u_{2}$ by solving the time reversed (31). Top-right: total field $u$. Bottom-right: discrepancy between $u$ and the sum of the recovered fields $u_{1}+u_{2}$. 


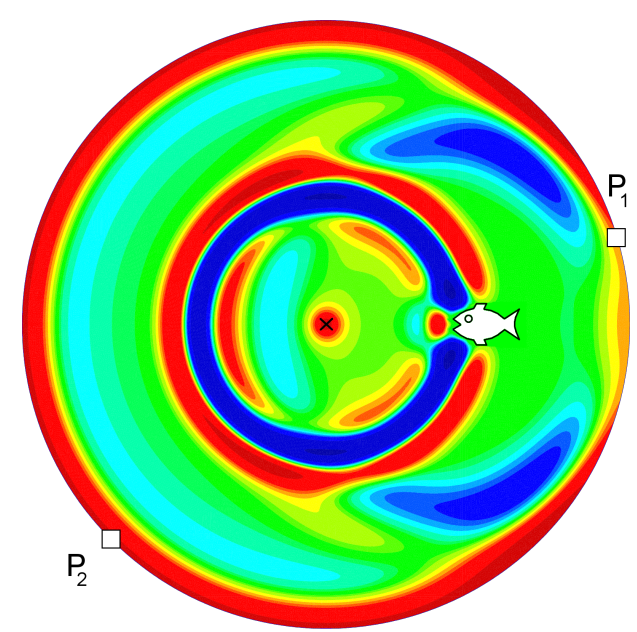

Figure 14: Scattering from a sound-soft inclusion. Snapshot of the total wave field at $t=0.73$.

knowing only approximately the origin of the scattered wave field from the obstacle. Again, the total field $u^{T}$ is recorded at $\Gamma$, the circle of radius $5 \lambda / 2$ centered at $C_{1}$.

In Fig. 15, we display the exact and the recovered wave fields located on $\Gamma$ at $P_{1}$ located at $\theta_{1}=\pi / 12$. Here, $u_{1}$ denotes the incident wave $u^{I}$, originating from the point source, and $u_{2}$ the scattered field $u^{S}$ due to the obstacle. In the top left frame, we compare $u_{1}$, obtained by solving (22), with the exact wave field and observe that both coincide quite well. Similarly, the wave field $u_{2}$, recovered by solving (31), compares favorably with the exact wave field emanating from $C_{2}$. Then in the top right frame, we compare the sum $u_{1}+u_{2}$ of the two recovered fields from (22) and (31), with the recorded total field $u$. Here we observe tiny errors in the reconstruction due to the first-order approximation in the progressive wave expansion (4). Nevertheless, when we add the recovered signals $u_{1}$ and $u_{2}$ and compare them to the recorded total field, the discrepancy is still smaller than $1 \%$ of the average amplitude. Although $u$ is small at $P_{1}$ due to the wave cancellation inside the shadow zone, we are still able to split it into the incident and scattered field, both larger in magnitude than the observed total field.

In Fig. 16, we show the time history of the recovered fields $u_{1}$ and $u_{2}$ at $P_{2}$ located at $\theta_{1}=5 \pi / 4$. Here, we solve the time reversed equation (27) to recover $u_{1}$, and the time reversed counterpart of (31) to obtain $u_{2}$. The observation point $P_{2}$ is now closer to the point source at $C_{1}$ than to the ob- 

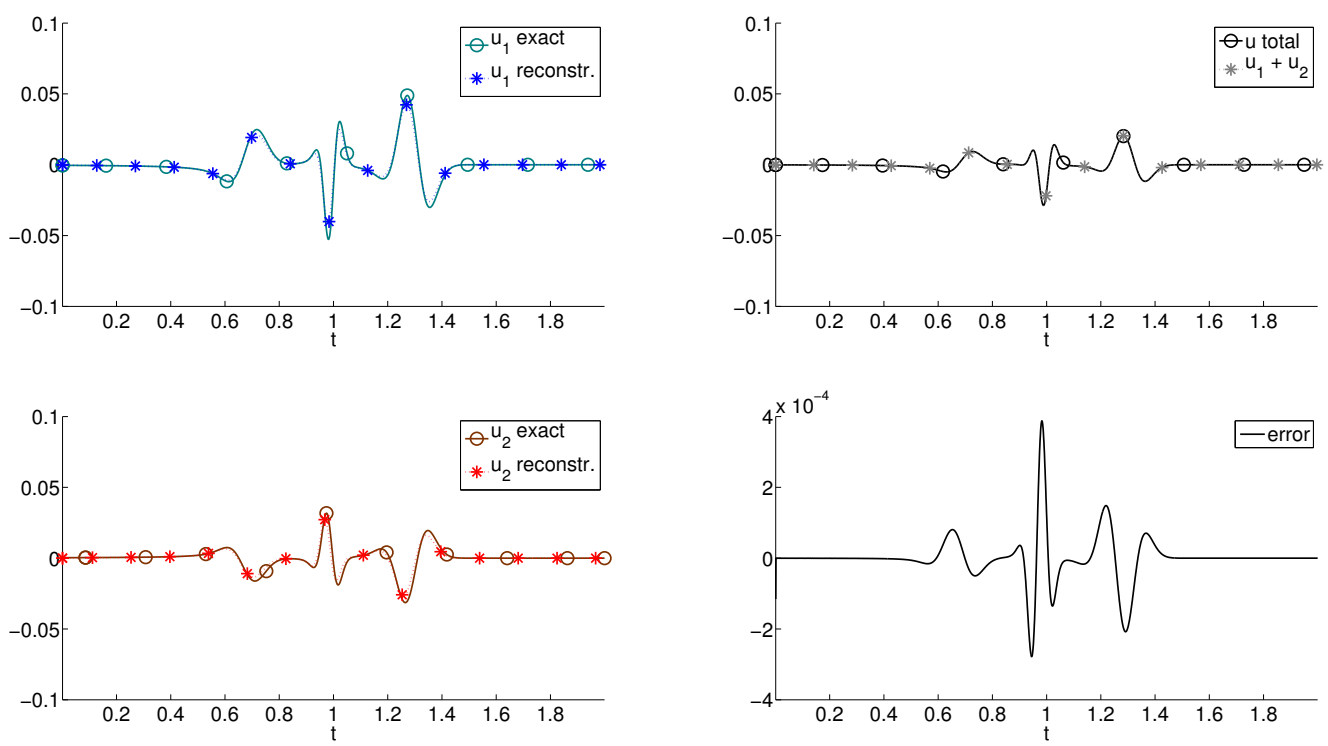

Figure 15: Scattering from a sound-soft inclusion. Both $u_{1}$ and $u_{2}$, recovered from total field measurements $u$, are shown at location $P_{1}$ vs. time. Top-left: recovery of $u_{1}=u^{I}$ by solving (22). Bottom-left: recovery of $u_{2}=u^{S}$ by solving (31). Top-right: total field $u=u^{T}$. Bottom-right: discrepancy between $u$ and the sum of the recovered fields $u_{1}+u_{2}$.
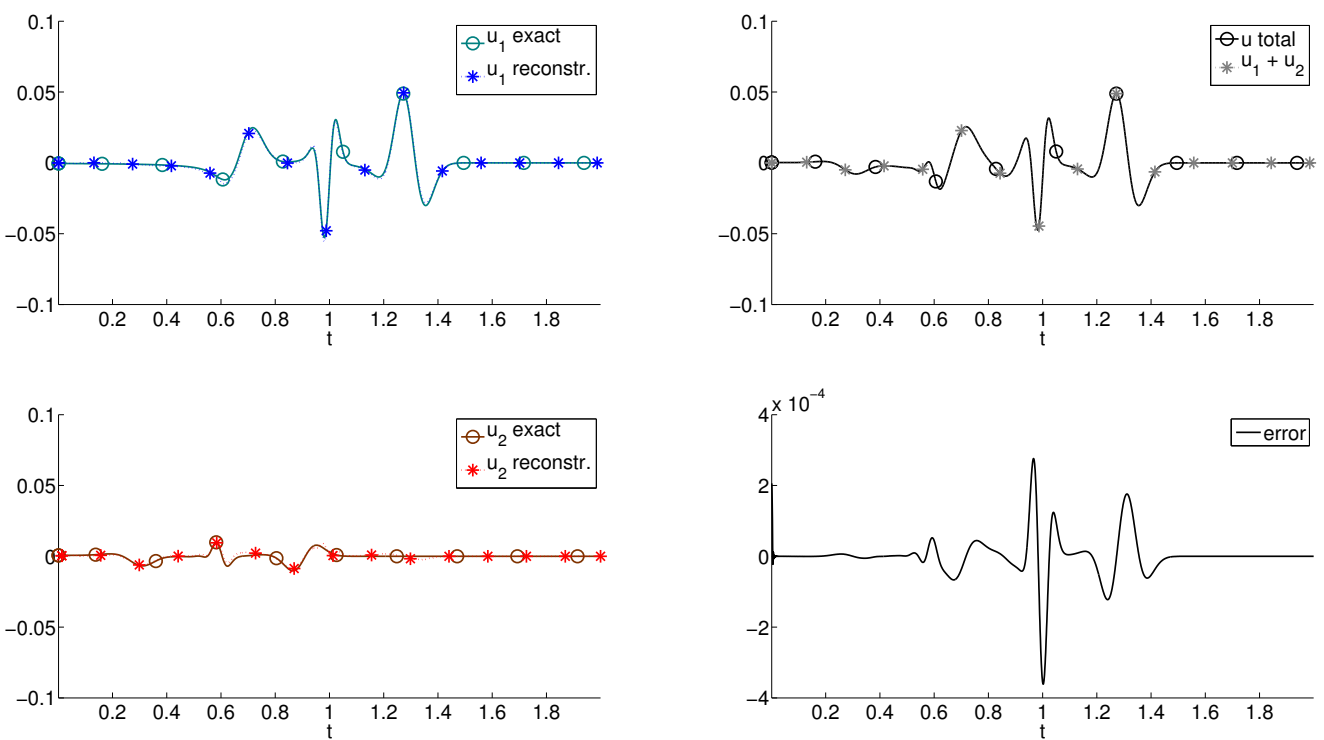

Figure 16: Scattering from a sound-soft inclusion. Both $u_{1}$ and $u_{2}$, recovered from total field measurements $u$, are shown at location $P_{2}$ vs. time. Top-left: recovery of $u_{1}=u^{I}$ by solving (27). Bottom-left: recovery of $u_{2}=u^{S}$ by solving the time reversed (31). Top-right: total field $u=u^{T}$. Bottom-right: discrepancy between $u$ and the sum of the recovered fields $u_{1}+u_{2}$. 
stacle. Again, the recovered outgoing wave fields $u_{1}$ and $u_{2}$ agree remarkably well with the exact wave fields. Although the incident field clearly dominates the scattered field in amplitude, we are still able to recover the time history of both wave fields.

\subsection{Noisy observations}

Finally, to verify the robustness of our wave splitting approach, we now add multiplicative noise to the observations as follows. Let

$$
N\left(t_{j}, x_{i}\right):=1+\sigma \xi_{i, j}
$$

where $x_{i}$ denotes the position of receiver $i, t_{j}$ denotes the $j^{\text {th }}$ time step, $\xi_{i, j}=\mathcal{N}(0,1)$ is a centered reduced normally distributed random variable, and $\sigma$ is the noise level. Instead of regularizing the observations prior to differentiation in (22), (31), we directly multiply the right-hand side of (22) as

$$
\left[\alpha\left(\theta_{k}\right) \partial_{t}+\beta\left(\theta_{k}\right) \partial_{\theta_{k}}+\gamma\left(\theta_{k}\right)\right] f_{k}=N \times g(u) .
$$

In Fig. 17, we performed our wave splitting approach on a perturbed right-hand-side in the case of two point sources. We apply 50\% Gaussian

noise to the right-hand-side as described in (38) and display the exact and the recovered wave fields on $\Gamma$ for $\theta_{1}=13 \pi / 12$. Again, the time history of fields $u_{1}$ and $u_{2}$ is recovered with small perturbations. As before, we also display the sum of the two recovered wave fields and observe that it is also little perturbed - see zoom in Fig. 18. In summary, our wave splitting approach seems remarkably robust with respect to added noise.

\section{Concluding remarks}

Starting from classical absorbing boundary conditions, we have devised a method to split a time-dependent, scattered, total wave field $u$ into its distinct outgoing components, $u_{i}$, induced by separate sources or obstacles. In doing so, we have shown that every $u_{i}$ satisfies a hyperbolic partial differential equation on the submanifold $\Gamma$, where total field measurements are assumed available. Depending on the particular geometry considered, the resulting initial-boundary value problem for $u_{i}$ on any subset of $\Gamma$ is well-posed either forward or backward in time. In two space dimensions, for instance, when $\Gamma$ lies on a circle, it is given by (22), (27) for the scattered field component induced by any source or obstacle at the center of $\Gamma$. Since it involves only 

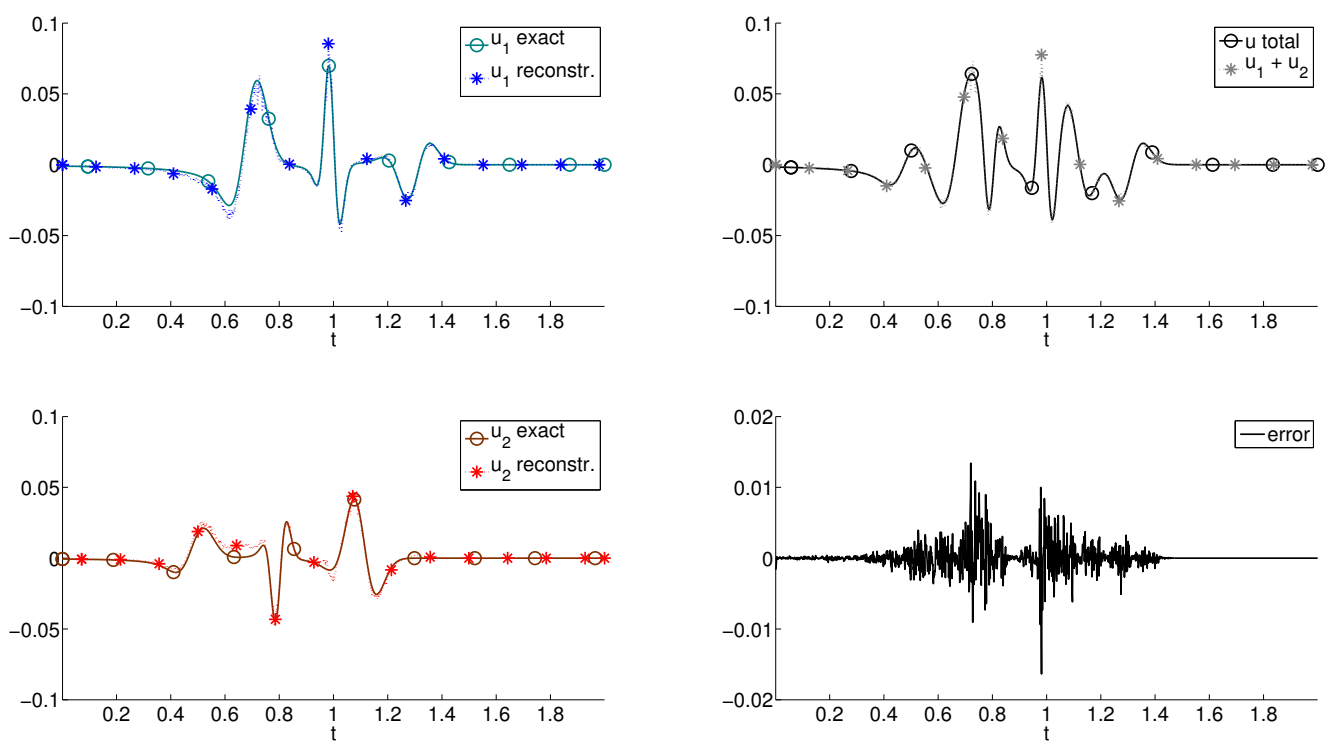

Figure 17: Noisy observations. Both $u_{1}$ and $u_{2}$, recovered from total field measurements $u$ with $50 \%$ noise, are shown at location $P_{2}$ vs. time. Top-left: recovery of $u_{1}$ by solving (27). Bottom-left: recovery of $u_{2}$ by solving the time reversed (31). Top-right: total field $u$. Bottom-right: discrepancy between $u$ and the sum of the recovered fields $u_{1}+u_{2}$.
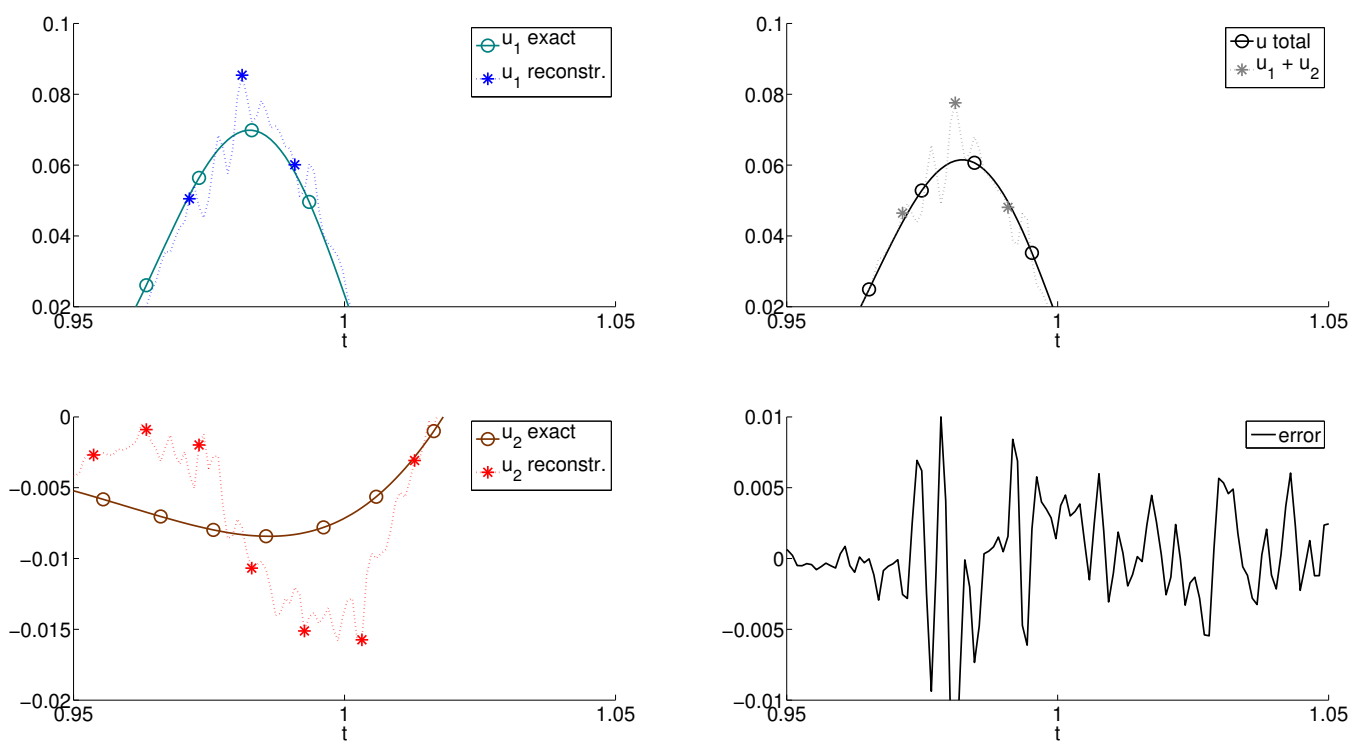

Figure 18: Noisy observations. Zoom of the recovery of wave fields $u_{1}$ and $u_{2}$ from total field measurements $u$ with $50 \%$ noise, are shown at location $P_{2}$ vs. time. Top-left: zoom of the recovery of $u_{1}$ by solving (27). Bottom-left: zoom of the recovery of $u_{2}$ by solving the time reversed (31). Top-right: total field $u$. Bottom-right: discrepancy between $u$ and the sum of the recovered fields $u_{1}+u_{2}$. 
time or tangential derivatives of $u_{i}$ at $\Gamma$, our approach is truly local in space and time. In fact, it is even possible to recover the scattered field components $u_{i}$ only over a part of the boundary $\Gamma$, as long as $u$ is known there too.

The derivation presented here in detail for two space dimensions, immediately extends to three space dimensions. In fact, we then expect even higher accuracy from the first-order Bayliss-Turkel condition (5), because the progressive wave expansion (3) now truly converges at finite distance while higher order corrections decay even faster with distance. Clearly, higher order absorbing boundary conditions, or possibly their counterparts without high-order derivatives $[18,19,11,6]$, could be used for higher accuracy, if needed. In particular, in the presence of three or more obstacles, higher order boundary conditions are probably needed for splitting the scattered fields. Our wave splitting approach also immediately applies to vector-valued wave equations, such as from electromagnetics and elasticity, for which local absorbing boundary conditions are known [22, 13, 28].

Once any scattered field component $u_{i}$ has been extracted from the total wave field, the corresponding inverse problem can be solved separately to recover the individual scatterer. Thus, the computational domain will be smaller in size and the inverse problem less ill-conditioned than the original multiple scattering inverse problem. Even further reduction of the computational domain can be achieved by back-propagating numerically $u_{i}$ towards the scatterer using the TRAC method [10], which will also yield an additional noise reduction in the data [4].

When the different components of the total wave field are not due to separate sources or scatterers, yet propagate at different speeds, such as shear and pressure waves in elasticity, our wave splitting approach probably also applies. Thus by applying appropriate radiation boundary conditions $[22,28]$ to the total elastic wave field, we could separate shear from pressure wave and thereby improve, for instance, full-field elasticity imaging [3].

\section{Acknowledgements}

This work was partially supported by the Swiss National Science Fondation. 


\section{References}

[1] S. Acosta. On-surface radiation condition for multiple scattering of waves. Comput. Meth. Appl. Mech. Engrg., 283:1296-1309, 2015.

[2] R. Aichner, H. Buchner, F. Yan, and W. Kellermann. A real-time blind source separation scheme and its application to reverberant and noisy acoustic environments. Signal Processing, 86(8):1260-1277, 2006.

[3] H. Ammari, E. Bretin, J. Garnier, W. Jing, H. Kang, and A. Wahab. Localization, stability, and resolution of topological derivative based imaging functionals in elasticity. SIAM J. Imaging Sci., 6(4):2174-2212, 2013.

[4] F. Assous, M. Kray, F. Nataf, and E. Turkel. Time Reversed Absorbing Condition: Application to inverse problem. Inverse Problems, 27(6):065003, 2011.

[5] A. Bayliss and E. Turkel. Radiation boundary conditions for wave-like equations. Comm. Pure Appl. Math., 33(6):707-725, 1980.

[6] E. Bécache, D. Givoli, and T. Hagstrom. High-order absorbing boundary conditions for anisotropic and convective wave equations. J. Comput. Phys., 229(4):1099-1129, 2010.

[7] A. Belouchrani, K. Abed-Meraim, J.-F. Cardoso, and E. Moulines. A blind source separation technique using second-order statistics. Signal Processing, IEEE Trans., 45(2):434-444, 1997.

[8] F. Ben Hassen, J. Liu, and R. Potthast. On source analysis by wave splitting with applications in inverse scattering of multiple obstacles. $J$. Comput. Math, 25(3):266-281, 2007.

[9] M. Castella, P. Bianchi, A. Chevreuil, and J.-C. Pesquet. A blind source separation framework for detecting CPM sources mixed by a convolutive MIMO filter. Signal Processing, 86(8):1950-1967, 2006.

[10] M. de Buhan and M. Kray. A new approach to solve the inverse scattering problem for waves: combining the TRAC and the Adaptive Inversion methods. Inverse Problems, 29(8):085009, 2013. 
[11] D. Givoli, T. Hagstrom, and I. Patlashenko. Finite element formulation with high-order absorbing boundary conditions for time-dependent waves. Comput. Methods Appl. Mech. Engrg., 195(2932):3666-3690, 2006.

[12] R. Griesmaier, M. Hanke, and J. Sylvester. Far field splitting for the Helmholtz equation. SIAM J. Numer. Anal., 52(1):343-362, 2014.

[13] M. J. Grote. Local nonreflecting boundary condition for Maxwell's equations. Comput. Meth. Appl. Mech. Engrg., 195(29-32):3691-3708, 2006.

[14] M. J. Grote and C. Kirsch. Dirichlet-to-Neumann boundary conditions for multiple scattering problems. J. Comput. Phys., 201(2):630-650, 2004.

[15] M. J. Grote and C. Kirsch. Nonreflecting boundary condition for timedependent multiple scattering. J. Comput. Phys., 221(1):41-67, 2007.

[16] M. J. Grote, M. Kray, F. Nataf, and F. Assous. Wave splitting for timedependent scattered field separation. Comptes Rendus Mathematiques, 353(6):523-527, 2015.

[17] M. J. Grote and I. Sim. Local nonreflecting boundary condition for time-dependent multiple scattering. J. Comput. Phys., 230(8):31353154, 2011.

[18] T. Hagstrom and S. I. Hariharan. A formulation of asymptotic and exact boundary conditions using local operators. Applied Numerical Mathematics, 27:403-416, 1998.

[19] T. Hagstrom and T. Warburton. A new auxiliary variable formulation of high-order local radiation boundary conditions: corner compatibility conditions and extensions to first-order systems. Wave Motion, 39(4):327-338, 2004.

[20] S. Haykin and Z. Chen. The cocktail party problem. Neural Computation, 17(9):1875-1902, 2005.

[21] F. Hecht. New development in FreeFem++. J. Numer. Math., 20(34):251-265, 2012. 
[22] R. L. Higdon. Radiation boundary conditions for elastic wave propagation. SIAM J. Numer. Anal., 27(4):831869, 1990.

[23] A. L. Klibanov, P. T. Rasche, M. S. Hughes, J. K. Wojdyla, K. P. Galen, J. H. J. Wible, and G. H. Brandenburger. Detection of Individual Microbubbles of Ultrasound Contrast Agents: Imaging of Free-Floating and Targeted Bubbles. Investigative Radiology, 39(3):187-195, 2004.

[24] K. L. Kreider. A wave splitting approach to time dependent inverse scattering for the stratified cylinder. SIAM J. Appl. Math., 49(3):932943, 1989 .

[25] M. S. Pedersen, D. Wang, J. Larsen, and U. Kjems. Two-microphone separation of speech mixtures. IEEE Transactions on Neural Networks, 19(3):475-492, 2008.

[26] M. Pernot, G. Montaldo, M. Tanter, and M. Fink. Ultrasonic stars for time reversal focusing using induced cavitation bubbles. Appl. Phys. Lett., 88(3):034102, 2006.

[27] R. Potthast, F. M. Fazi, and P. A. Nelson. Source splitting via the point source method. Inverse Problems, 26(4):045002, 2010.

[28] D. Rabinovich, D. Givoli, J. Bielak, and T. Hagstrom. A finite element scheme with a high order absorbing boundary condition for elastodynamics. Comput. Methods Appl. Mech. Engrg., 200(23-24):2048-2066, 2011.

[29] T. Saratoon, T. Tarvainen, B. T. Cox, and S. R. Arridge. A gradientbased method for quantitative photoacoustic tomography using the radiative transfer equation. Inverse Problems, 29(7):075006, 19, 2013.

[30] V. Twersky. On multiple scattering of waves. J. Res. Nat. Bur. Standards, 64D:715-730, 1960.

[31] J. G. Tylka and E. Y. Choueiri. Comparison of techniques for binaural navigation of higher-order ambisonic soundfields. AES 139 Convention, page 9421, 2015.

[32] V. H. Weston. Factorization of the wave equation in higher dimensions. J. Math. Phys., 28:1061-1068, 1987. 